\title{
The Effect of Umbellularia californica Essential Oil on Blood Vessel Diameter in Frogs
}

\author{
Holden M. Wagstaff, Stephan R. Maman, Mary Jo Tufte, \& Matthew S. Weeg* \\ Department of Biology, Southern Utah University, Cedar City, UT \\ Students: holden.wagstaff@gmail.com,stephanmaman@gmail.com \\ Mentors: tuftem@suu.edu,matthewweeg@suu.edu*
}

\begin{abstract}
Plant essential oils contain many chemicals that are physiologically active in vertebrates. Terpenoids, which represent the largest category of these compounds, have been shown to lower blood pressure by reducing cardiac output and causing vasodilation. Most studies on the vasoactivity of terpenoids have been done on aortic rings and mesenteric artery preparations, and little is known about their effects on microvasculature. We applied Umbellularia californica essential oil, which contains several different terpenoids, to the cutaneous microvasculature of frogs and measured changes in blood vessel diameter. The U. californica oil caused a sustained, reversible vasoconstriction of these blood vessels, whereas no significant change in diameter was caused by medical grade sesame oil, which served as our control. The vasoconstriction we observed was therefore induced by compounds in the $U$. californica oil, many of which have previously been shown to cause vasodilation in arteries. Our results suggest that the vasoactive effects of terpenoids may differ across blood vessel types, and are more variable than previously thought.
\end{abstract}

\section{KEYWORDS}

Cardiovascular; Terpenoid; Vasoactive; Arteriole; Secondary Metabolite; Vasoconstriction; Essential Oil; Microvasculature

\section{INTRODUCTION}

Most plant species produce secondary metabolites that, among other things, provide protection against herbivory. ${ }^{1}$ Although these compounds largely have negative physiological effects on herbivores, ${ }^{2}$ many of them have been investigated for potential medicinal uses and exhibit promising therapeutic effects. ${ }^{3}$ The most ubiquitous secondary metabolites are the terpenoids, of which over 40,000 have been identified. ${ }^{4}$ Intravenous administration of terpenoids reduces blood pressure, ${ }^{5-8}$ which is thought to occur because these compounds reduce heart rate ${ }^{5,7-10}$ and cause vasodilation. ${ }^{6-11}$ These findings have raised the possibility that terpenoids may prove effective at treating hypertension. ${ }^{12}$

Whether terpenoids universally cause vasodilation throughout the cardiovascular system is unknown. Studies on their vascular effects have been conducted almost exclusively on mammalian aortic rings and mesenteric arteries, ${ }^{13}$ and little is known about how these compounds affect the microvasculature and veins. There is evidence to suggest that curcumin, a terpenoid that causes vasorelaxation in arteries, ${ }^{14,15}$ can cause vasoconstriction in arterioles. ${ }^{16}$ This raises the possibility that terpenoids are not exclusively vasodilators, and that their effects may vary depending on blood vessel type.

To explore the vasoactive effects of terpenoids on microvasculature, we applied Umbellularia californica essential oil to cutaneous blood vessels of frogs. Frog skin is particularly well suited for this purpose because it is highly vascularized with dense beds of epidermal capillaries and networks of subcutaneous arterioles and venules. ${ }^{17}$ These subcutaneous vessels are readily accessible by surgical resection of the skin, ${ }^{18}$ allowing microscopic changes in blood vessel diameter to be measured following treatment with vasoactive chemicals. We chose $U$. californica essential oil because it contains a cocktail of terpenoids, ${ }^{19}$ several of which induce vasorelaxation in isolated aortic rings and/or mesenteric arteries. ${ }^{9},{ }^{20-23}$ Our objective for this study was to determine whether essential oil containing these compounds causes a similar response in the microvasculature as well.

\section{METHODS AND PROCEDURES}

Essential oil distillation

Aerial portions of Umbellularia californica were collected near San Francisco, CA, and dried at room temperature. Essential oil was obtained by steam distillation ${ }^{24}$ and stored in the dark at room temperature. GC-MS analysis of the oil verified the presence of the terpenoids umbellulone, eucalyptol, and terpinen-4-ol (H. Kansagra, personal communication). 


\section{Experimental procedure}

Experimental methods were approved by SUU IACUC (2013302-S). Fifteen leopard frogs (Rana pipiens) were obtained from Connecticut Valley Biological Supply Company. Frogs were double-pithed and covered with moist paper towels to maintain cutaneous respiration. Because anesthetics are known to alter the physiology of the amphibian cardiovascular system, ${ }^{25,26}$ frogs were not anesthetized before the pithing procedure. Subcutaneous blood vessels were exposed by making incisions in the skin covering the limbs and pinning it flat on a dissection tray. The exposed blood vessels were bathed in amphibian Ringer's solution until the time of treatment. Regions that were well supplied with vessels in which blood flow could be observed were treated with $10 \mu \mathrm{l}$ of either U. californica oil or medical grade sesame oil (Sigma-Aldrich; St. Louis, MO, USA). Sesame oil was used as a control to ensure that changes in blood vessel diameter were due to the chemical components of the U. californica oil rather than potential hydrophobic effects of the oil. Only one site per limb was treated, with multiple limbs used on each frog.

To record changes to blood vessel diameter, digital photos were captured before and after oil application with an eyepiecemounted digital camera (Moticam 10; Motic, Richmond, BC, Canada) and image capturing software (Motic Images Plus 2.0; Motic). One image was captured prior to oil application to give a baseline measure of blood vessel diameter. Images were then captured once per second for 300 seconds after oil application, and imported into NIH ImageJ for subsequent data analysis.

\section{Data collection}

To measure vasoactive effects of the oil, we compared blood vessel diameter before and 60 seconds after treatment. Six blood vessels were randomly selected from each pre-treatment image. Data measurement tools in Image J were used to measure the diameter of these vessels in each pair of before and after treatment images. Blood vessels were selected and measurements were taken without knowledge of the type of oil that had been applied. Anatomical landmarks such as chromatophores were used to ensure that each vessel was measured at the same point along its length in each image. Pre- and post-treatment measurements were taken from 102 vessels in the experimental group and 84 vessels in the control group.

Data analysis

All data are presented as mean +/- standard error. Paired t-tests were used to determine whether U. californica essential oil and/or sesame oil caused a significant change in blood vessel diameter. Unpaired t-tests were used to determine whether there was a significant difference in the percent change in diameter between U. californica essential oil and sesame oil.

\section{RESULTS}

Application of $U$. californica essential oil caused several blood vessels at each site to become noticeably smaller in diameter, while other blood vessels did not appear to be affected (Figure 1). U. californica essential oil caused a significant decrease in blood vessel diameter, $t(99)=6.63, p=1.75 \times 10^{-9}$, as the average diameter before treatment was $46.1 \mu \mathrm{m}+/-3.4$ and after treatment was 32.2 $\mu \mathrm{m}+/-2.7$ (Figure 2). This effect persisted for the duration of data acquisition (300 seconds post-treatment), but was reversed when the oil was washed off with Ringer's solution (data not shown). There were no visible changes in the diameter of any blood vessels following application of our sesame oil control. Data analysis confirmed that sesame oil did not have significant vasoactive effects, $t(83)=-1.34, p=0.18$, as the average vessel diameter before treatment was $49.9 \mu \mathrm{m}+/-4.2$ and after treatment was 50.7 $\mu \mathrm{m}+/-4.4$ (Figure 2). The percent change in blood vessel diameter caused by U. californica essential oil $(-28.9 \%+/-3.1)$ was significantly greater, $t(140)=-8.71, p=7.8 \times 10^{-15}$, than that caused by our sesame oil control $(1.1 \%+/-1.5$; Figure 3). 

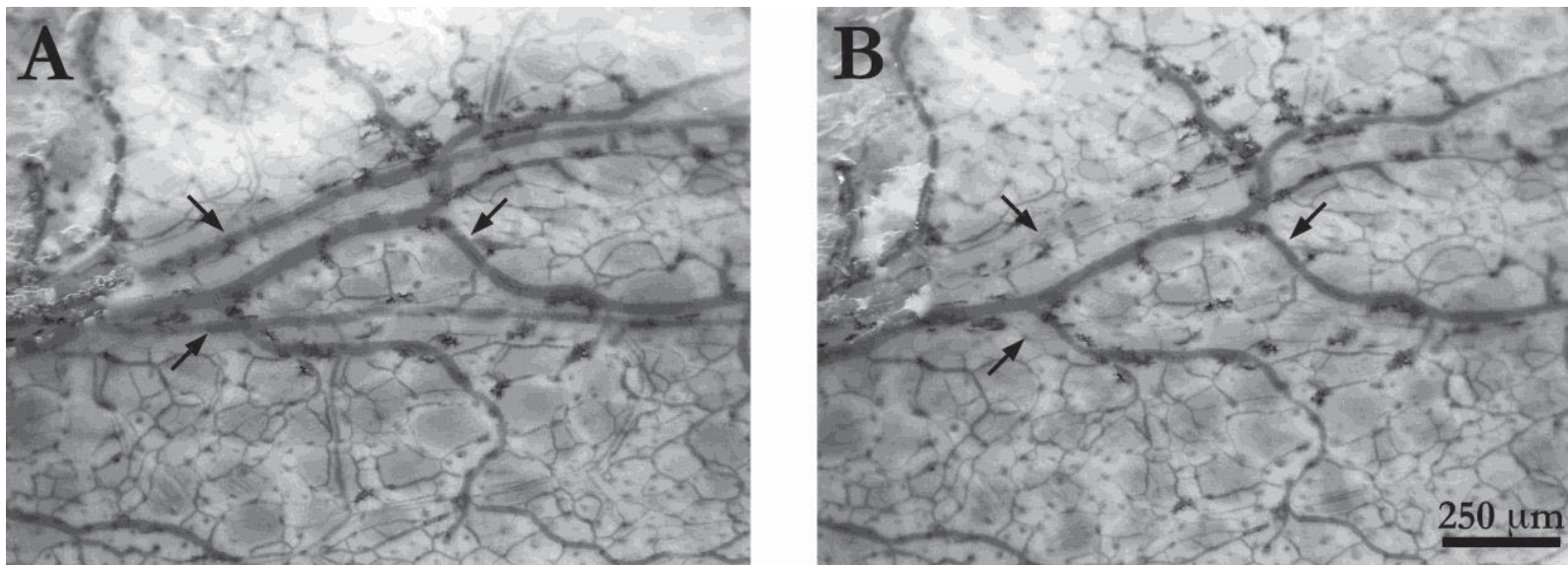

Figure 1. Photomicrographs of cutaneous blood vessels before $(\mathbf{A})$ and 60 seconds after $(\mathbf{B})$ application of $10 \mu \mathrm{l}$ of $U$. californica essential oil. U. californica essential oil caused vasoconstriction in many blood vessels (arrows). Scale bar $=250 \mu \mathrm{m}$.

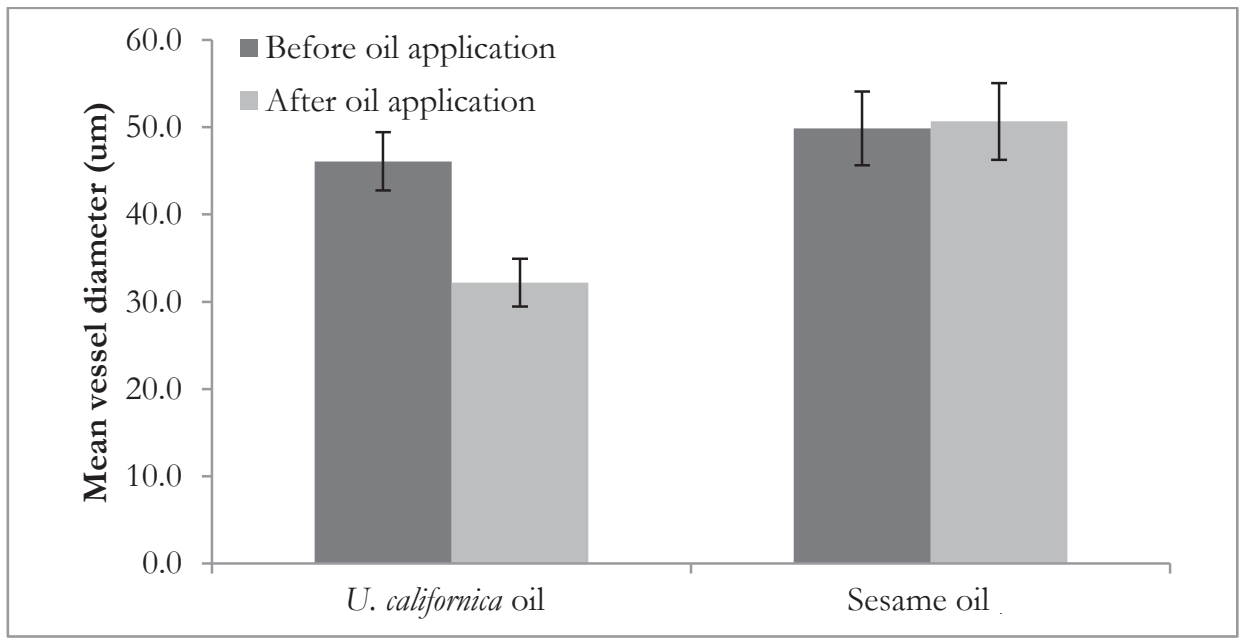

Figure 2. Mean cutaneous blood vessel diameter before and after application of $10 \mu \mathrm{l} U$. californica essential oil or medical grade sesame oil. U. californica essential oil caused a significant decrease in vessel diameter $\left(p=1.75 \times 10^{-9}\right.$, paired t-test), while sesame oil caused no significant change in vessel diameter $(p=0.18)$. Error bars represent standard error. 


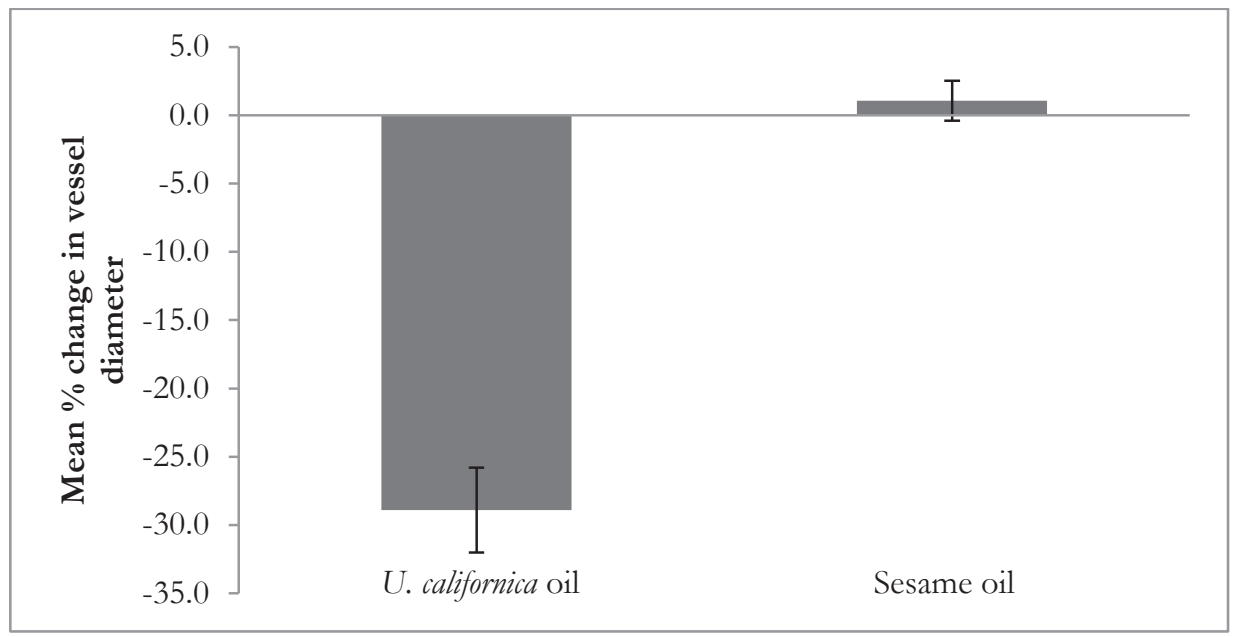

Figure 3. Mean percent change in cutaneous blood vessel diameter caused by application of $10 \mu l$ U. californica essential oil or medical grade sesame oil. U. californica essential oil caused a significantly greater change in blood vessel diameter than sesame oil $\left(p=4.2 \times 10^{-14}\right.$, unpaired t-test). Error bars represent standard error.

\section{DISCUSSION}

Our results indicate that $U$. californica essential oil causes significant vasoconstriction when applied directly to cutaneous blood vessels in frogs. Because our control did not affect blood vessel diameter, we conclude that the vasoconstriction we observed was due to the vasoactive properties of one or more components of the $U$. californica oil rather than its hydrophobic nature. The return of the blood vessels to their original diameter after washing with Ringer's solution indicates that the change in diameter was physiological, and not due to any unknown cytotoxic effects of the oil.

We observed considerable variation in the degree of vasoconstriction caused by U. californica oil, with some vessels constricting almost completely and some not at all. It is possible that cutting and pinning the skin compromised the integrity of some vessels and not others, leading to the differences we observed. However, we only applied oil to regions in which blood was flowing in all of the vessels, which suggests that they were all physiologically viable. More likely, these results arose from the different types of blood vessels present in frog skin and the specific vasoactive properties of each vessel type. The subcutaneous layer of amphibian skin contains arterioles that supply epidermal capillary beds and venules that drain them. Blood flow through the capillaries is controlled primarily by changes in the diameter of arterioles, ${ }^{27}$ which have a stronger vasoactive response than venules. ${ }^{28} \mathrm{We}$ therefore hypothesize that the vessels showing the greatest vasoconstriction were arterioles and those showing little or no response were venules.

The vasoconstriction that we observed is contrary to the vasodilatory effects of terpenoids on aortic rings and mesenteric arteries. ${ }^{6-11}$ Our results are also inconsistent with the hypotensive effects that occur when terpenoids are administered systemically, ${ }^{5-8}$ as a drop in blood pressure suggests arteriolar vasodilation rather than vasoconstriction. These differences are surprising, given that our oil was comprised primarily of the terpenoids umbellulone, 1,8-cineole (eucalyptol), and terpinen-4-ol, which have all been shown to cause arterial vasodilation. ${ }^{12,13}$ Umbellulone causes an increase in meningeal blood flow, which is interpreted as vasodilation, when administered intravenously or intranasally. ${ }^{29}$ This occurs due to an interaction between umbellulone and trigeminal neurons rather than vascular smooth muscle, which may explain the difference in vascular response. 1,8-cineole (eucalyptol) causes vasodilation when applied directly to aortic rings or mesenteric arteries, ${ }^{9,20}$ and intravenous injections of terpinen-4-ol cause a decrease in blood pressure that is thought to arise from vasodilation. ${ }^{30}$ Thus, our results are contrary to all previously published work on the vasoactivity of compounds contained within U. californica oil.

There are a number of possible explanations for these differences. Previous studies on the vasoactive effects of terpenoids have focused on mammals, so our results may reflect species-specific effects. It would be interesting to apply U. californica oil to a mammalian preparation such as the rabbit cheek pouch to investigate this hypothesis. Many vasoactive compounds interact synergistically, ${ }^{31-33}$ and it is possible that vasoconstriction arises from the combined activity of the terpenoids within $U$. californica essential oil. This idea could be explored by applying umbellulone, eucalyptol, and terpinen-4-ol individually and in different combinations to our frog skin preparation. We are most intrigued by the possibility that our results reflect differences in how arteries and arterioles respond to terpenoids. This hypothesis is supported by studies on the terpenoid curcumin, which causes 
vasodilation when applied to isolated aortic rings, ${ }^{14}$ but vasoconstriction when applied at high concentrations to cutaneous arterioles. ${ }^{16}$ The physiological cause of this difference is unknown, but may be due to differences in the receptors found on arterial and arteriolar smooth muscle. This possibility warrants further investigation, including applying U. californica oil to arteries or applying known vasodilatory terpenoids to microvasculature.

\section{CONCLUSIONS}

Previous research has supported the hypothesis that terpenoids act exclusively as vasodilators. ${ }^{12,13}$ However, the majority of studies have been conducted on arteries, with little attention paid to arterioles. Our results lend support to the alternative hypothesis that the vasoactive effects of terpenoids are variable, and that vessels of the microvasculature respond differently to these compounds than arteries do. Future research should focus on the microvasculature to evaluate these competing hypotheses, and determine the physiological mechanisms behind the variability in terpenoid effects.

\section{ACKNOWLEDGEMENTS}

The authors thank T. Hildebrandt for bringing U. californica to our attention and H. Kansagra for GC-MS analysis. D. Tufte and J. Grant provided help with statistics and feedback on an earlier version of this manuscript. We also thank two anonymous reviewers for their thoughtful feedback. Funding was generously provided by SUU UGRASP, an L.S. \& Aline W. Skaggs Research Fellowship, and a Walter Maxwell Gibson Research Fellowship.

\section{REFERENCES}

1. Fürstenberg-Hägg, J., Zagrobelny, M., and Bak, S. (2013) Plant defense against insect herbivores, Int J Mol Sci 14, $10242-10297$.

2. Iason, G. (2005) The role of plant secondary metabolites in mammalian herbivory: ecological perspectives, Proc Nutr Soc 64, $123-131$.

3. Edris, A.E. (2007) Pharmaceutical and therapeutic potentials of essential oils and their individual volatile constituents: a review, Phytother Res 21, 308-323.

4. Aharoni, A., Jongsma, M.A., and Bouwmeester, H.J. (2005) Volatile science? Metabolic engineering of terpenoids in plants, Trends Plant Sci 10, 594-602.

5. Aydin, Y., Kutlay, Ö., Ari, S., Duman, S., Uzuner, K., and Aydin, S. (2007) Hypotensive effects of carvacrol on the blood pressure of normotensive rats, Planta Med 73, 1365-1371.

6. Bastos, J.F., Moreira, Í.J., Ribeiro, T.P., Medeiros, I.A., Antoniolli, A.R., De Sousa, D.P., and Santos, M.R. (2010) Hypotensive and vasorelaxant effects of citronellol, a monoterpene alcohol, in rats, Basic Clin Pharmacol Toxicol 106, 331-337.

7. Guedes, D.N., Silva, D.F., Barbosa-Filho, J.M., and Medeiros, I.A. (2002) Muscarinic agonist properties involved in the hypotensive and vasorelaxant responses of rotundifolone in rats, Planta Med 68, 700-704.

8. Interaminense, L.F.L., Jucá, D.M., Magalhães, P.J.C., Leal-Cardoso, J.H., Duarte, G.P., and Lahlou, S. (2007) Pharmacological evidence of calcium-channel blockade by essential oil of Ocimum gratissimum and its main constituent, eugenol, in isolated aortic rings from DOCA-salt hypertensive rats, Fundam Clin Pharmacol 21, 497-506.

9. Lahlou, S., Figueiredo, A.F., Magalhães, P.J.C., and Leal-Cardoso, J.H. (2002) Cardiovascular effects of 1, 8-cineole, a terpenoid oxide present in many plant essential oils, in normotensive rats, Can J Physiol Pharmacol 80, 1125-1131.

10. Ribeiro, T.P., Porto, D.L., Menezes, C.P., Antunes, A.A., Silva, D.F., De Sousa, D.P., Nakao, L.S., Braga, V.A., and Medeiros, I.A. (2010) Unravelling the cardiovascular effects induced by $\alpha$-terpineol: a role for the nitric oxide-cGMP pathway, Clin Exp Pharmacol Physiol 37, 811-816.

11. Criddle, D.N., Madeira, S.V.F., and Moura, R.S. (2003) Endothelium-dependent and-independent vasodilator effects of eugenol in the rat mesenteric vascular bed, J Pharm Pharmacol 55, 359-365.

12. Santos, M.R., Moreira, F.V., Fraga, B.P., De Souza, D.P., Bonjardim, L.R., and Quintans-Junior, L.J. (2011) Cardiovascular effects of monoterpenes: a review, Rev Bras Farmacogn 21, 764-771.

13. Luna-Vázquez, F.J., Ibarra-Alvarado, C., Rojas-Molina, A., Rojas-Molina, I., and Zavala-Sánchez, M.Á. (2013) Vasodilator compounds derived from plants and their mechanisms of action, Molecules 18, 5814-5857.

14. Sasaki, Y., Goto, H., Tohda, C., Hatanaka, F., Shibahara, N., Shimada, Y., Terasawa, K., and Komatsu, K. (2003) Effects of curcuma drugs on vasomotion in isolated rat aorta, Biol Pharm Bull 26, 1135-1143.

15. Xu, P.-H., Long, Y., Dai, F., and Liu, Z.-L. (2007) The relaxant effect of curcumin on porcine coronary arterial ring segments, V ascul Pharmacol 47, 25-30.

16. Dewar, A.M., Clark, R.A., Singer, A.J., and Frame, M.D. (2011) Curcumin mediates both dilation and constriction of peripheral arterioles via adrenergic receptors, J Invest Dermatol 131, 1754-1760.

17. Czopek, J. (1965) Quantitative studies on the morphology of respiratory surfaces in amphibians, Cells Tissues Organs 62, 296323.

18. Pinder, A., Clemens, D., and Feder, M. (1990) An isolated perfused frog skin preparation for the study of gas exchange, in Oxygen Transport to Tissue XII (Piiper, J., Goldstick, T., and Meyer, M., Ed.) 719-724, Springer, New York. 
19. Tabanca, N., Avonto, C., Wang, M., Parcher, J.F., Ali, A., Demirci, B., Raman, V., and Khan, I.A. (2013) Comparative investigation of Umbellularia californica and Laurus nobilis leaf essential oils and identification of constituents active against Aedes aegypti, J Agric Food Chem 61, 12283-12291.

20. Pinto, N.V., Assreuy, A.M.S., Coelho-de-Souza, A.N., Ceccatto, V.M., Magalhães, P.J.C., Lahlou, S., and Leal-Cardoso, J.H. (2009) Endothelium-dependent vasorelaxant effects of the essential oil from aerial parts of Alpinia zerumbet and its main constituent 1, 8-cineole in rats, Phytomedicine 16, 1151-1155.

21. Lahlou, S., Interaminense, L.F.L., Magalhães, P.J.C., Leal-Cardoso, J.H., and Duarte, G.P. (2004) Cardiovascular effects of eugenol, a phenolic compound present in many plant essential oils, in normotensive rats, J Cardiovasc Pharmacol 43, $250-257$.

22. Peixoto-Neves, D., Silva-Alves, K.S., Gomes, M.D.M., Lima, F.C., Lahlou, S., Magalhães, P.J.C., Ceccatto, V.M., Coelho-deSouza, A.N., and Leal-Cardoso, J.H. (2010) Vasorelaxant effects of the monoterpenic phenol isomers, carvacrol and thymol, on rat isolated aorta, Fundam Clin Pharmacol 24, 341-350.

23. Magalhães, P.J.C., Lahlou, S., Jucá, D.M., Coelho-de-Souza, L.N., Frota, D., Tibúrcio, P.T., Da Costa, A.M.G., and LealCardoso, J.H. (2008) Vasorelaxation induced by the essential oil of Croton nepetaefolius and its constituents in rat aorta are partially mediated by the endothelium, Fundam Clin Pharmacol 22, 169-177.

24. Starmans, D.A., and Nijhuis, H.H. (1996) Extraction of secondary metabolites from plant material: a review, Trends Food Sci Technol 7, 191-197.

25. Cakir, Y., and Strauch, S.M. (2005) Tricaine (MS-222) is a safe anesthetic compound compared to benzocaine and pentobarbital to induce anesthesia in leopard frogs (Rana pipiens), Pharmacol Rep 57, 467-474.

26. Goulet, F., Hélie, P., and Vachon, P. (2010) Eugenol anesthesia in African clawed frogs (Xenopus laevis) of different body weights, J Am Assoc Lab Anim Sci 49, 460-463.

27. Segal, S.S. (2005) Regulation of blood flow in the microcirculation, Microcirculation 12, 33-45.

28. Malvin, G.M., and Walker, B.R. (2001) Sites and ionic mechanisms of hypoxic vasoconstriction in frog skin, Am J Physiol-Regul Integr Comp Physiol 280, R1308-R1314.

29. Nassini, R., Materazzi, S., Vriens, J., Prenen, J., Benemei, S., Siena, G.D., la Marca, G., Andrè, E., Preti, D., Avonto, C., Sadofsky, L., Marzo, V.D., Petrocellis, L.D., Dussor, G., Porreca, F., Taglialatela-Scafati, O., Appendino, G., Nilius, B., and Geppetti, P. (2011) The "headache tree" via umbellulone and TRPA1 activates the trigeminovascular system, Brain 135, 376390.

30. Lahlou, S., Galindo, C.A., Leal-Cardoso, J.H., Fonteles, M.C., and Duarte, G.P. (2002) Cardiovascular effects of the essential oil of Alpinia zerumbet leaves and its main constituent, Terpinen-4-ol, in rats: role of the autonomic nervous system, Planta Med 68, 1097-1102.

31. Gardiner, S.M., Compton, A.M., Bennett, T., Kemp, P.A., and Ney, U. (1990) Synergistic internal carotid vasodilator effects of human $\alpha$-calcitonin gene-related peptide and nimodipine in conscious rats, Br J Pharmacol 99, 830-834.

32. Li, J., and Meng, Z. (2009) The role of sulfur dioxide as an endogenous gaseous vasoactive factor in synergy with nitric oxide, Nitric Oxide 20, 166-174.

33. Oliveira, M.A., Fortes, Z.B., Santos, R.A., Kosla, M.C., and De Carvalho, M.H.C. (1999) Synergistic effect of angiotensin-(1-

7) on bradykinin arteriolar dilation in vivo, Peptides 20, 1195-1201.

\section{ABOUT THE STUDENT AUTHORS}

Holden Wagstaff graduated with a Bachelor of Science in Biology from Southern Utah University in 2015. He is currently attending the University of Rochester School of Medicine and Dentistry seeking an MD degree.

Stephan R. Maman graduated with a Bachelor of Science in Biology from Southern Utah University in 2015. He is currently pursuing an MD degree at Penn State College of Medicine and will graduate in May 2019.

\section{PRESS SUMMARY}

Essential oils contain chemicals that can alter the physiology of animals. Many of these chemicals affect the cardiovascular system by increasing the diameter of large arteries. We applied Umbellularia californica essential oil to smaller arterioles and observed a decrease in diameter, opposite to what was expected. Our results suggest that arterioles respond differently to the chemicals in essential oils, and that the effect of essential oils on the cardiovascular system is more variable than previously described. 